\title{
Relationship of Idiopathic Osteonecrosis of the Femoral Head to Perfusion Changes in the Proximal Femur by Dynamic Contrast-Enhanced MRI
}

\author{
Wing P. Chan ${ }^{1,2}$ \\ Yi-Jui Liu ${ }^{3}$ \\ Guo-Shu Huang 4 \\ Min-Fang Lin ${ }^{2}$ \\ Sydney Huang ${ }^{2,5}$ \\ Yue-Cune Chang ${ }^{6}$ \\ Ching-Chuan Jiang ${ }^{5}$
}

Keywords: bone marrow, dynamic contrast-enhanced MRI, femur, osteonecrosis, perfusion

DOI:10.2214/AJR.10.4322

Received January 21, 2010; accepted after revision June 28,2010

This work was supported by the Department of Health, Taiwan, Republic of China (grants NHRI-GT-EX88-E820L, NHRI-GT-EX89-E820L, and NHRI-EX90-8820EL).

'Department of Radiology, School of Medicine, Taipei Medical University, Taipei, Taiwan, Republic of China.

${ }^{2}$ Department of Radiology, Taipei Medical UniversityWan Fang Hospital, Taipei, Taiwan, Republic of China.

${ }^{3}$ Department of Automatic Control Engineering, Feng Chia University, Taichung, Taiwan, Republic of China.

${ }^{4}$ Department of Radiology, Tri-Service General Hospital, National Defense Medical Center, Taipei, Taiwan, Republic of China.

${ }^{5}$ Department of Orthopedic Surgery, National Taiwan University Hospital, 7 Chung San S. Rd., Taipei 100, Taiwan, Republic of China. Address correspondence to C.C. Jiang (ccj@ccms.ntu.edu.tw).

${ }^{6}$ Department of Mathematics, Tamkang University, Tamsui, Taipei, Taiwan, Republic of China.

AJR2011; 196:637-643

0361-803X/11/1963-637

(c) American Roentgen Ray Society

OBJECTIVE. The purpose of this article is to relate intramedullary perfusion of the proximal femur to severity of osteonecrosis of the femoral head by using dynamic contrastenhanced MRI (DCE-MRI).

SUBJECTS AND METHODS. Twelve patients (14 symptomatic hips) who underwent DCE-MRI and had subsequent core decompression of the femoral head were examined. Hips were graded for severity according to MRI findings and were assigned scores of 0 (negative findings), 1 (focal marrow abnormalities), and 2 (subchondral collapse). Thirteen asymptomatic hips acted as controls. The DCE-MRI data were analyzed by use of a pharmacokinetic twocompartment model.

RESULTS. Compared with control hips, there was significantly greater peak enhancement in the femoral head in hips of all grades $(p<0.001)$ and in the femoral neck $(p=0.001)$ and intertrochanteric area $(p=0.001)$ in grade 2 hips. The time to peak was significantly delayed in the femoral head in grade 0 hips $(p=0.02)$ and in the intertrochanteric area in grade 2 hips ( $p=0.003)$ compared with the controls.

CONCLUSION. As evaluated by DCE-MRI, intramedullary peak enhancement in the femoral head increased with progression of idiopathic osteonecrosis of the femoral head, whereas there was delayed peak enhancement in the femoral head in hips with negative findings and intertrochanteric stasis in advanced osteonecrosis of the femoral head. Such perfusion changes as shown on MRI can occur with early osteonecrosis in the absence of other MRI abnormalities.

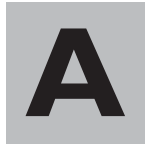
lthough the pathogenesis of nontraumatic osteonecrosis of the femoral head is poorly understood, alteration of intramedullary circulation is known to be involved. Ficat and Arlet [1] and Ficat [2] observed with intramedullary venography that rapid clearance of contrast medium by efferent vessels occurs in the normal hip, but reflux into the diaphysis leads to intramedullary stasis in hips with osteonecrosis of the femoral head.

Dynamic contrast-enhanced MRI (DCEMRI) has been used to evaluate blood flow of bone marrow in vivo [3-6]. Most studies have used a posttraumatic model of induced osteonecrosis of the femoral head in animals. In human subjects, Bluemke et al. [7] first reported greater enhancement in the femoral head, but lesser enhancement in the femoral neck, in patients with systemic lupus erythematosus than in healthy subjects, as assessed by DCEMRI. Malizos et al. [8] first reported delayed peak enhancement in hips with acute bone marrow edema $40-65$ seconds after a first pass of gadolinium-based contrast agent; Aaron et al. [9] also observed similar findings as analyzed with pharmacokinetic modeling.

This study aims to relate intramedullary blood perfusion of the proximal femur to the severity of osteonecrosis of the femoral head by using DCE-MRI. We hypothesized, first, that blood flow stasis occurs in the proximal femur in the early stage of osteonecrosis of the femoral head because of perfusion reflux into the diaphysis; second, that when osteonecrosis of the femoral head progresses in severity, blood perfusion increases in the femoral head because of increasing granulation tissue; and finally, that these perfusion changes can be evaluated with DCE-MRI.

\section{Subjects and Methods \\ Patients}

The study was approved by our institution's review board, and written informed consent was obtained from patients. We studied 27 consecutive patients with clinically suspected osteonecrosis of the femoral head who underwent DCE-MRI and 
had subsequent intramedullary pressure measurement and core decompression or total hip arthroplasty. One orthopedic surgeon examined all patients in outpatient clinics before ordering MRI. All patients had experienced typical rest pain of the affected hips for 4-24 months before the MRI studies. The pattern was dull aching or throbbing pain in the groin or anterior thigh. The duration of osteonecrosis of the femoral head could not be tracked precisely. No patient had a history of autoimmune disease, rheumatic disease, or sickle cell anemia. Of these 27 patients, 13 who had a history of trauma and two who had suboptimal MRI studies were excluded.

Finally, 12 patients (14 symptomatic hips) with suspected nontraumatic osteonecrosis of the femoral head who underwent DCE-MRI and had subsequent core decompression of the femoral head were recruited. All patients denied risk factors such as alcoholism or steroid use and were therefore categorized as having idiopathic osteonecrosis. The asymptomatic hips of 10 patients were used as controls. We also recruited three asymptomatic hips in three additional patients who had one painful hip and received DCE-MRI only (without decompression surgery).

One orthopedic surgeon measured the intramedullary pressure of all the symptomatic hips by use of a method described elsewhere [1, 2]. A baseline pressure of $30 \mathrm{~mm} \mathrm{Hg}$ or higher at the femoral head was considered abnormal [1]. The intramedullary pressure of the contralateral asymptomatic hips and the hips of the three additional patients was not measured. Three patients with four affected hips had follow-up MRI 5-9 months after core decompression. The follow-up study used the same imaging protocol as those on the initial examination and was performed in the same MRI unit.

\section{MRI}

MRI was performed on a 0.5 -T scanner (Vectra, GE Healthcare). With patients in a supine position, both hip joints were imaged simultaneously with use of a circumferential body coil. The routine initial protocol was as follows: unenhanced coronal spin-echo T1-weighted (TR/TE, 450/20) and STIR (2,500/25; inversion time, 100 milliseconds) images, axial conventional spin-echo T2-weighted $(4,000 / 100)$ images, and sagittal gradient-echo (320/20; flip angle, $30^{\circ}$ ) images. Three coronal scans, determined from an axial scout view, were obtained at 3- and 5-mm intervals for precise localization. A coronal image passing through the femoral heads and necks bilaterally on a single plane was selected for DCEMRI study. At the time that bolus IV injection of $0.2 \mathrm{~mL} / \mathrm{kg}$ gadopentetate dimeglumine (Magnevist, Bayer HealthCare) by autoinjector began, a dynamic T1-weighted (300/25) sequence was obtained at a 16-second acquisition time for each image for the next 7 minutes after the scan started. The rate of injection was fixed at $2.0 \mathrm{~mL} / \mathrm{s}$ via a 23 -gauge catheter in an antecubital vein; a $20-\mathrm{mL}$ saline flush was administered at the same flow rate immediately after the gadolinium injection. In total, 29 dynamic data sets were obtained. Other imaging parameters included a $40-\mathrm{cm}$ field of view, a $256 \times 256$ matrix, a 5-mm section thickness, and 1 excitation.

\section{Data Analysis}

Two radiologists, each with at least 15 years of experience in skeletal MRI, interpreted all MRI scans without knowledge of the clinical data, and the final interpretations were reached by consensus. Hips were divided into four groups. The control group included symptom-free hips with negative MRI findings. The symptomatic hips formed the other three groups. In the grade 0 group, the affected hips had elevated intramedullary pressure but no focal marrow MRI abnormalities in the femoral head. Although these patients underwent core decompression to treat their symptoms, initially a grade 0 hip was not considered clinically to have osteonecrosis of the femoral head. The grade 1 group had focal marrow MRI abnormalities (such as bands, rings, lines, or subchondral crescents) in the affected femoral head [1012], indicating early osteonecrosis of the femoral head. In the grade 2 group, there was subchondral collapse of the femoral head in addition to focal marrow abnormalities, indicating an advanced stage of osteonecrosis of the femoral head.
Any diffuse marrow abnormalities in the femoral head extending to the intertrochanteric area were also evaluated; typically, these were hypointense on T1-weighted images and hyperintense on T2weighted or STIR images [13].

The data obtained from each DCE-MRI study were transferred to a PC and processed by software developed in-house by using Matlab (MathWorks). We used a pharmacokinetic two-compartment Brix model to analyze the DCE-MRI data [14]. The model assumes that the relative signal change is linear, proportional to the concentration of gadopentetate dimeglumine. On the basis of this model, the signal-time curve can be described relative to the signal intensity of the tissue after bolus injection of gadopentetate dimeglumine by the following mathematic formula:

$$
\frac{S(t)-S_{0}}{S_{0}}=\frac{A}{\left(k_{21}-k_{e l}\right)}\left(\exp \left[-k_{e l} t\right]-\exp \left[k_{21} t\right]\right)(1),
$$

where $S(t)$ is the time signal after administration of gadopentetate dimeglumine, $S_{0}$ is the unenhanced signal, $A$ is the amplitude of uptake (i.e., plasma concentration of gadopentetate dimeglumine), $k_{21}$ is the exchange rate (i.e., exchange of gadopentetate dimeglumine between extracellular space and the plasma), and $k_{e l}$ is the washout rate (i.e., excretion of gadopentetate dimeglumine by the kidneys). One operator measured signal intensity within the boundaries of the region of interest (Fig. 1), containing 120-130 pixels, in the femoral heads and necks, intertrochanteric areas, and acetabula. The fitted signal-time curve of the region of interest, whose signal was an average of all pixels, was calculated by using this equation with nonlinear least-
Fig. 1-Coronal T1-weighted MRI shows operatordefined regions of interest (numbered ovals) over bilateral hips (top to bottom: acetabulum, femoral head, femoral neck, and intertrochanteric area). Signal measured was obtained from average of all pixels within each region of interest. Same measurement was also made on dynamic contrast-enhanced MRI.

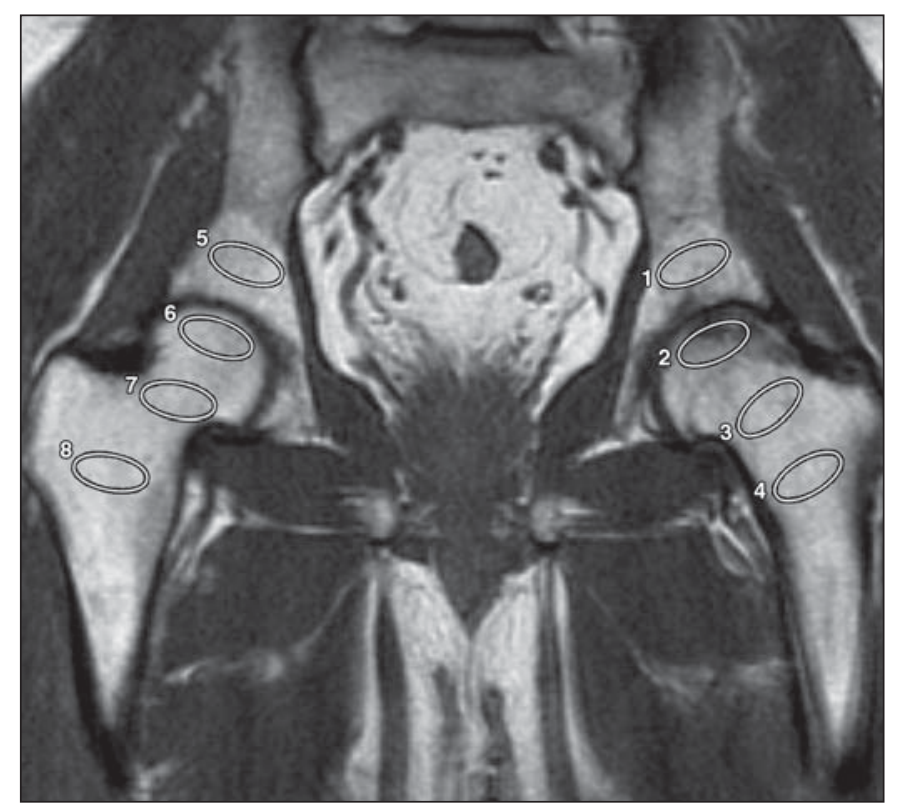




\section{Dynamic Contrast-Enhanced MRI of Femoral Idiopathic Osteonecrosis}

TABLE I: Percentage Peak of Enhancement in Various Femoral Sites in Hips With Symptoms of Osteonecrosis of the Femoral Head Versus Control Hips

\begin{tabular}{l|c|c|c|c|r}
\hline \multirow{2}{*}{\multicolumn{1}{c|}{ Site }} & \multicolumn{5}{|c}{ Grade of Osteonecrosis of Femoral Head } \\
\cline { 2 - 6 } & $\begin{array}{c}\text { Control } \\
(n=13 \mathrm{Hips})\end{array}$ & $\begin{array}{c}\text { Grade 0 } \\
(n=3 \mathrm{Hips})\end{array}$ & $\begin{array}{c}\text { Grade 1 } \\
(n=5 \mathrm{Hips})\end{array}$ & $\begin{array}{c}\text { Grade 2 } \\
(n=6 \mathrm{Hips})\end{array}$ & $p^{\mathrm{a}}$ \\
\hline Femoral head & $0.23 \pm 0.06$ & $0.51 \pm 0.36^{\mathrm{b}}$ & $1.37 \pm 1.01^{\mathrm{b}}$ & $2.05 \pm 1.07^{\mathrm{b}}$ & $<0.001$ \\
Femoral neck & $0.37 \pm 0.21$ & $0.83 \pm 0.54$ & $0.90 \pm 0.50$ & $4.26 \pm 1.62^{\mathrm{b}}$ & 0.001 \\
Intertrochanteric area & $0.38 \pm 0.12$ & $0.70 \pm 0.20^{\mathrm{b}}$ & $1.91 \pm 2.71$ & $2.23 \pm 0.86^{\mathrm{b}}$ & 0.001 \\
Acetabulum & $0.21 \pm 0.09$ & $0.15 \pm 0.07$ & $0.19 \pm 0.10$ & $0.19 \pm 0.07$ & 0.778 \\
\hline
\end{tabular}

Note-Data are mean \pm SD normalized ratio of the specific femoral site to the acetabulum.

aKruskal-Wallis test.

${ }^{\mathrm{b}} p<0.05$ versus control. The $p$ values of each of three outcome measures were multiplied by 3 for Bonferroni corrections of multiple comparisons.

square-error curve fitting. In this study, the peak enhancement value, calculated as $\left(S_{\max }-S_{0}\right) / S_{0}$ of the fitted signal-time curve for each patient was computed for evaluating femoral marrow perfusion. Each DCE-MRI value was normalized to the acetabulum before statistical analysis.

\section{Statistical Analysis}

To assess differences in perfusion parameters (such as peak signal, amplitude of uptake, time to peak, exchange rate, and washout rate) among the four groups, we used Kruskal-Wallis tests. The corresponding post hoc multiple comparisons, to compare perfusion parameters at various femoral sites in each symptomatic group versus the control group, were performed with Mann-Whitney tests with Bonferroni corrections for multiple comparisons. All the analyses were done with SPSS software (version 15.0, SPSS). All tests were two-tailed, and a $p$ value of less than 0.05 was considered to indicate a statistically significant difference.

\section{Results}

There were eight women and four men, with a mean $( \pm$ SD) age of $45 \pm 10.4$ years. Five patients were affected on the right side and five were affected on the left side. Two patients had bilateral hip pain. All patients underwent MRI examination within 4 weeks before measurement of intramedullary pressure and surgery. The remaining 10 contralateral hips were unequivocally symptom free and negative on MRI. These 12 patients with 14 affected hips and 10 control hips and the three additional control patients are the subject of this report.

Focal bone marrow abnormalities were noted in 11 femoral heads - in five grade 1 hips (two subchondral rings, one bandlike pattern, one subchondral line, and one crescent) and in six grade 2 hips (two bandlike patterns and four subchondral crescents). Diffuse marrow abnormalities were detected in two proximal femurs. Three were grade 0 (no abnormalities). All affected hips had abnormally elevated intramedullary pressure (range, 52-128 mm Hg) in the femoral head.

Quantitative analysis revealed gradual increases in peak signal intensity in the femoral head with progression in the severity of osteonecrosis of the femoral head $(p<0.01)$. Peak signal intensity was higher in the intertrochanteric area in grade 0 hips $(p=0.02)$ and in the femoral neck $(p=0.001)$ and intertrochanteric area $(p=0.001)$ in grade 2 hips (Table 1; Fig. 2). There were no significant differences in peak enhancement in the acetabulum among the groups $(p>0.05)$. for four groups. $\mathrm{N}$ = controls.

A, Graph shows values for femoral heads.
The average amplitude of uptake had statistical results similar to those for the peak signal for all femoral sites in grade 1 and 2 osteonecrosis of the femoral head (Table 2).

There was significant delay in time-topeak values in the femoral head in grade 0 hips $(p=0.02)$ and in the intertrochanteric area in grade 2 hips $(p=0.003)$ compared with the control group (Table 3). Although there was no significant difference from control hips, all femoral sites in grade 1 and 2 hips had delayed time-to-peak values.

The exchange rate was statistically significantly lower in the intertrochanteric area in grade 2 hips than in controls $(p=0.01)$ (Table 4), whereas the washout rate did not show any statistically significant difference from control in any femoral sites in all grades of osteonecrosis of the femoral head $(p>0.05)$.

Follow-up MRI examinations showed progression of grades in the four affected hips (Figs. 3 and 4), including two grade 0 hips that progressed to grade 1 .

\section{Discussion}

The results of our study showed an increase in peak enhancement in the femoral head with progression of idiopathic osteonecrosis of the femoral head, as evaluated with DCE-MRI. Symptomatic hips

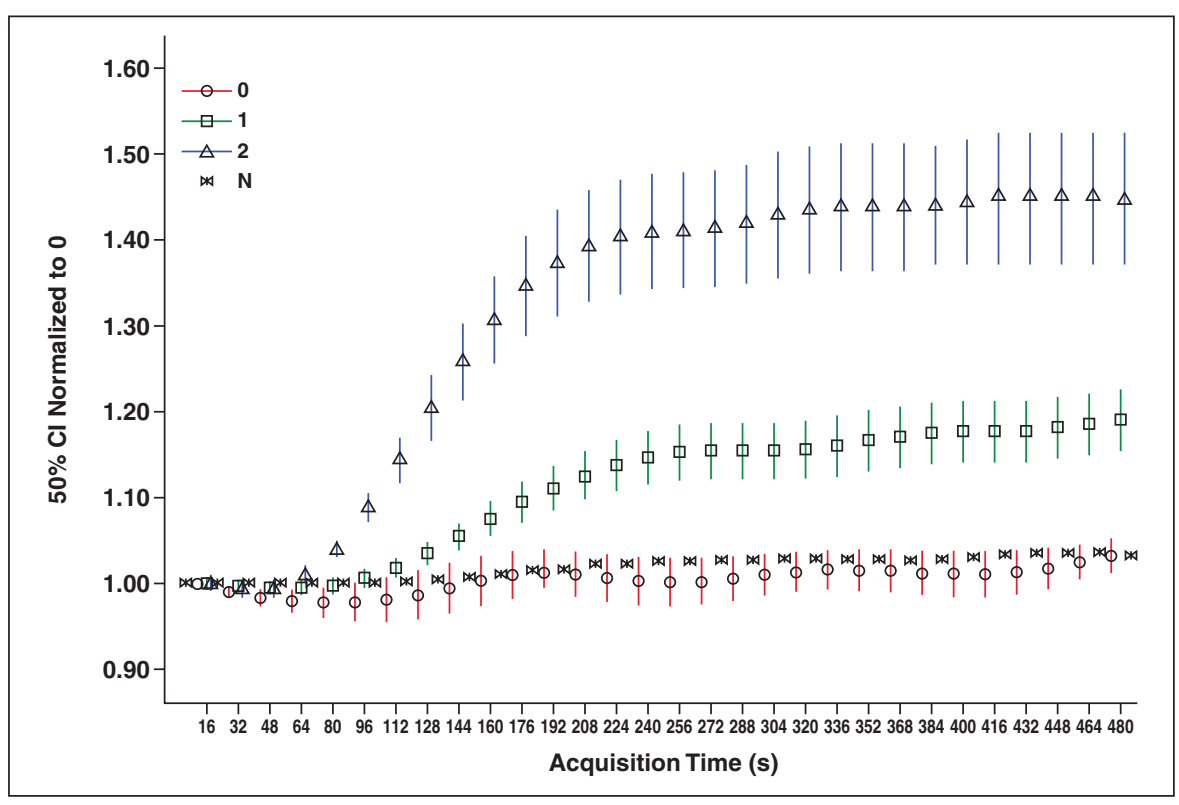

Fig. 2-Representative time-signal intensity curves from dynamic contrast-enhanced MRI of symptomatic versus control hips. Error bar plots represent $50 \%$ Cls for mean (peak enhancement normalized to acetabulum)

(Fig. 2 continues on next page) 

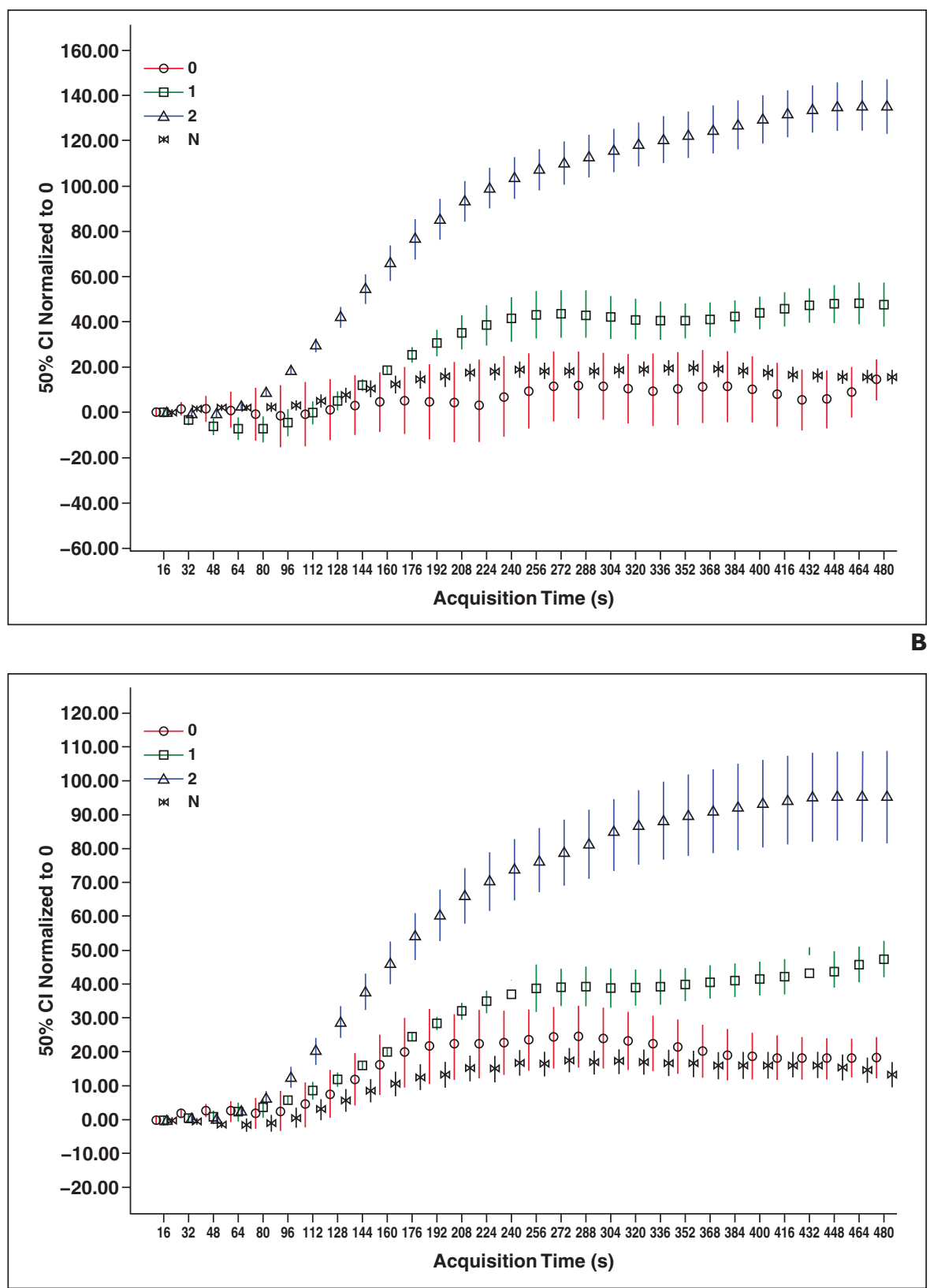

Fig. 2 (continued)-Representative time-signal intensity curves from dynamic contrast-enhanced MRI of symptomatic versus control hips. Error bar plots represent $50 \% \mathrm{Cls}$ for mean (peak enhancement normalized to acetabulum) for four groups. $\mathrm{N}=$ controls.

B, Graph shows values for femoral necks.

C, Graph shows values for intertrochanteric areas.

with elevated intramedullary pressure but no other abnormalities on MRI (grade 0) had delayed time-to-peak enhancement in the femoral head compared with the control hips, and two of these hips progressed to early osteonecrosis of the femoral head over the course of 9 months. This finding suggests a trend of decreased blood perfusion but increased blood volume in the fem-
With the pharmacokinetic model, it is possible to extract information on blood flow that appears to be consistent with the values obtained from DCE-MRI in hips with osteonecrosis of the femoral head. Peak signal enhancement is proportional to the concentration of gadolinium, and that enhancement is not directly related to perfusion, because perfusion can be reduced in vascular stasis but delayed peak enhancement can be increased.

The initial phase of ischemia relates to contraction of arteries and decrease in blood flow. Later, reactive hyperemia with vasodilation, increased blood flow, and interstitial edema most likely occurs [15]. Arterial dilatation is a reactive phenomenon after temporary ischemia. Yamano et al. [16] reported that the arteries supplying the femoral head are dilated in transient bone marrow edema syndrome. During hyperemia, intramedullary pressure is increased, and the patient experiences hip pain, as in the grade 0 hips in our study. The delayed time-to-peak value in grade 0 hips in the femoral head could be due to reactive hyperemia with vasodilatation and could be a way of detecting occult osteonecrosis of the femoral head.

In the femoral head, the diagnosis of osteonecrosis is based on detection of reparative reactions to necrotic tissue by MRI. Previous reports have indicated that most of the gadolinium enhancement on MRI is attributable to focal inflammatory infiltrates, fibrocytic repair [3], and viable granulation tissue [17-19] surrounding the necrotic areas. The greatest enhancement was found in the inner zone, with viable fibrous mesenchymal tissue with dilated vessels and interstitial edema, together with the low-signal outer zone, corresponding to the "double-line sign" $[15,19$, 20]. These histologic findings reported previously can explain most of our DCE-MRI results, such as gradual increase in peak enhancement of the femoral head with progression of the severity of osteonecrosis of the femoral head.

The baseline signal intensity may influence the peak measurement in early and advanced osteonecrosis, especially in the femoral head. In our study, patients with grade 1 and grade 2 hips had much lower baseline signal intensity on T1-weighted images than did those with grade 0 hips and the control hips. The peak signal increase caused by an increased concentration of gadolinium is also influenced by capillary leak of contrast material or tissue blood volume. This major contribution of baseline signal intensity to 


\section{Dynamic Contrast-Enhanced MRI of Femoral Idiopathic Osteonecrosis}

TABLE 2: Percentage Blood Volume (Amplitude of Uptake) in Various Femoral Sites in Hips With Symptoms of Osteonecrosis of Femoral Head Versus Control Hips

\begin{tabular}{l|c|c|c|c|c}
\hline \multirow{2}{*}{\multicolumn{1}{c|}{ Site }} & \multicolumn{5}{|c}{ Grade of Osteonecrosis of Femoral Head } \\
\cline { 2 - 6 } & $\begin{array}{c}\text { Control } \\
(n=13 \mathrm{Hips})\end{array}$ & $\begin{array}{c}\text { Grade 0 } \\
(n=3 \mathrm{Hips})\end{array}$ & $\begin{array}{c}\text { Grade 1 } \\
(n=5 \mathrm{Hips})\end{array}$ & $\begin{array}{c}\text { Grade 2 } \\
(n=6 \mathrm{Hips})\end{array}$ & $p^{\mathrm{a}}$ \\
\hline Femoral head & $0.24 \pm 0.21$ & $0.49 \pm 0.33$ & $0.77 \pm 0.46^{\mathrm{b}}$ & $1.75 \pm 0.80^{\mathrm{b}}$ & 0.001 \\
Femoral neck & $0.66 \pm 1.32$ & $1.02 \pm 0.53^{\mathrm{b}}$ & $0.72 \pm 0.68$ & $2.30 \pm 0.59^{\mathrm{b}}$ & 0.004 \\
Intertrochanteric area & $0.33 \pm 0.12$ & $0.41 \pm 0.17$ & $0.82 \pm 0.70$ & $1.25 \pm 0.51^{\mathrm{b}}$ & 0.007 \\
Acetabulum & $0.15 \pm 0.06$ & $0.15 \pm 0.05$ & $0.13 \pm 0.08$ & $0.14 \pm 0.07$ & 0.942 \\
\hline
\end{tabular}

Note-Data are mean \pm SD normalized ratio of the specific femoral site to the acetabulum.

${ }^{a}$ Kruskal-Wallis test.

${ }^{\mathrm{b}} p<0.05$ versus control. The $p$ values of each of three outcome measures were multiplied by 3 for Bonferroni corrections of multiple comparisons.

TABLE 3: Comparison of Time-to-Peak (Percentage per Minute) in Various Femoral Sites Between Symptomatic Versus Control Hips

\begin{tabular}{l|c|c|c|c|c}
\hline \multirow{2}{*}{\multicolumn{1}{c|}{ Site }} & \multicolumn{5}{|c}{ Grade of Osteonecrosis of Femoral Head } \\
\cline { 2 - 6 } & $\begin{array}{c}\text { Control } \\
(n=13 \mathrm{Hips})\end{array}$ & $\begin{array}{c}\text { Grade 0 } \\
(n=3 \mathrm{Hips})\end{array}$ & $\begin{array}{c}\text { Grade 1 } \\
(n=5 \text { Hips })\end{array}$ & $\begin{array}{c}\text { Grade 2 } \\
(n=6 \text { Hips })\end{array}$ & $p^{\mathrm{a}}$ \\
\hline Femoral head & $1.56 \pm 0.48$ & $2.65 \pm 0.95^{\mathrm{b}}$ & $1.78 \pm 0.46$ & $1.48 \pm 0.34$ & 0.065 \\
Femoral neck & $1.35 \pm 0.74$ & $1.77 \pm 1.75$ & $1.61 \pm 0.58$ & $1.57 \pm 0.24$ & 0.158 \\
Intertrochanteric area & $1.12 \pm 0.31$ & $1.97 \pm 1.56$ & $1.58 \pm 0.45$ & $1.61 \pm 1.17^{\mathrm{b}}$ & 0.024 \\
Acetabulum & $4.00 \pm 1.47$ & $2.84 \pm 0.86$ & $4.11 \pm 1.33$ & $4.13 \pm 0.55$ & 0.165 \\
\hline
\end{tabular}

Note-Data are mean \pm SD normalized ratio of the specific femoral site to the acetabulum.

${ }^{a}$ Kruskal-Wallis test.

${ }^{\mathrm{b}} p<0.05$ versus control. The $p$ values of each of three outcome measures were multiplied by 3 for Bonferroni corrections of multiple comparisons.

TABLE 4: Percentage Rate of Enhancement in Various Femoral Sites in Hips With Symptoms of Osteonecrosis of Femoral Head Versus Control Hips

\begin{tabular}{l|c|c|c|c|c}
\hline \multirow{2}{*}{\multicolumn{1}{c|}{ Site }} & \multicolumn{5}{|c}{ Grade of Osteonecrosis of Femoral Head } \\
\cline { 2 - 6 } \multicolumn{1}{c|}{} & $\begin{array}{c}\text { Control } \\
(n=13 \text { Hips })\end{array}$ & $\begin{array}{c}\text { Grade 0 } \\
(n=3 \text { Hips })\end{array}$ & $\begin{array}{c}\text { Grade 1 } \\
(n=5 \text { Hips })\end{array}$ & $\begin{array}{c}\text { Grade 2 } \\
(n=6 \text { Hips })\end{array}$ & $p^{\text {a }}$ \\
\hline Femoral head & $3.62 \pm 6.51$ & $3.83 \pm 2.58$ & $0.94 \pm 0.55$ & $0.71 \pm 0.21$ & 0.327 \\
Femoral neck & $2.48 \pm 4.20$ & $3.28 \pm 5.60$ & $1.78 \pm 1.36$ & $0.97 \pm 0.91$ & 0.579 \\
Intertrochanteric area & $1.34 \pm 1.58$ & $0.68 \pm 0.36$ & $1.59 \pm 2.29$ & $0.53 \pm 0.15^{\mathrm{b}}$ & 0.083 \\
Acetabulum & $0.43 \pm 0.39$ & $0.79 \pm 0.50$ & $0.29 \pm 0.09$ & $0.31 \pm 0.18$ & 0.180 \\
\hline
\end{tabular}

Note-Data are mean \pm SD normalized ratio of the specific femoral site to the acetabulum.

a Kruskal-Wallis test.

${ }^{\mathrm{b}} p<0.05$ versus control. The $p$ values of each of three outcome measures were multiplied by 3 for Bonferroni corrections of multiple comparisons.

enhancement changes explains, in part, the greater peak signal and amplitude of uptake in grade 1 and grade 2 hips than in grade 0 and control hips.

The peak enhancement ratio of bone marrow circulation is a complex process that takes into account blood inflow and outflow, vascular permeability, and the equilibrium of gadolinium between intravascular and interstitial spaces [21]. It is difficult to verify whether the mechanism of nontraumatic osteonecrosis of the femoral head begins on the mans because technically it should be compensated with a bolus injection of high-dose gadolinium so that a transient decrease in signal intensity can be detected [22].

T1-weighted DCE-MRI, as in our study, has been assumed to represent the venous distribution phases of gadolinium kinetics [22]. Bone marrow edema can retain gadolinium longer with greater volume, resulting in delayed peak enhancement that has not begun to resolve by 5 minutes of scan time. A similar phenomenon has been observed in hips with osteonecrosis of the femoral head. Aaron et al. [9] reported that the prolongation and enhancement of the outflow phase in patients with osteonecrosis of the femoral head can be interpreted as reflecting stasis (or decreased perfusion), most likely resulting from vascular obstruction, as preliminarily observed on intraosseous venography [1, 2]. In our study, similar findings were observed: delayed perfusion and an increase in exchange rate between extracellular space and the plasma in the intertrochanteric area, representing intramedullary stasis, in advanced osteonecrosis of the femoral head.

In addition to gadolinium's reflection of vascular stasis, enhancement is greater in tissue that contains more hematopoietic than fatty marrow [23-25]. Hematopoietic marrow has abundant sinusoidal spaces, whereas fatty marrow has a sparse vascular supply. The conversion of hematopoietic to fatty marrow correlates with physiologic decreases in intramedullary blood flow. Mitchell et al. [24] reported that only $13 \%$ of healthy individuals older than 50 years had intertrochanteric hematopoietic marrow. They observed an increase in fatty marrow and a concomitant decrease in hematopoietic marrow in a series of patients with osteonecrosis of the femoral head of various causes. Therefore, in our study, the contribution of hematopoietic marrow to peak enhancement could be negligible.

Decreased bone marrow perfusion can be associated with age and fatty marrow [26]. MRI shows that hips with osteonecrosis of the femoral head more often have predominantly fatty marrow in the intertrochanteric region of the femur [7, 21]. Marrow perfusion is inversely proportional to fat content [7]. Further animal studies may answer the question of whether the decreased perfusion of the femoral head in grade 0 hips, as in our study, is attributable predominantly to the intramedullary fat content.

The perfusion of the acetabulum did not differ with varying severity of osteonecrosis 


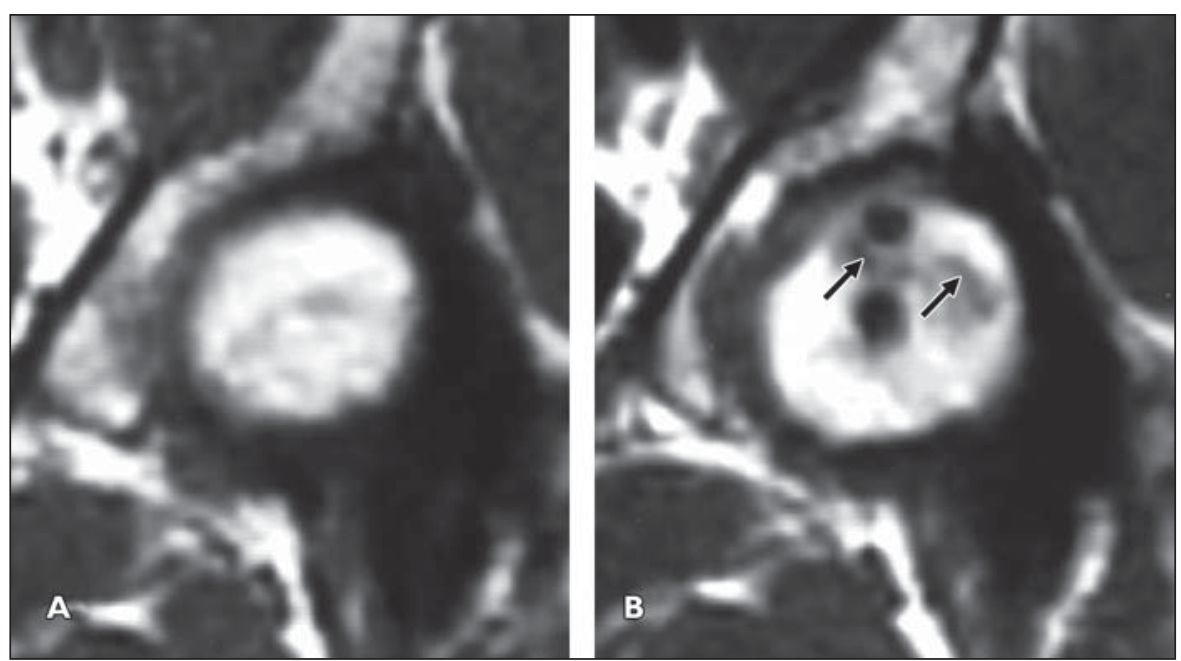

Fig. 3-47-year-old woman with osteonecrosis of femoral head with interval progression from stage 0 to stage 1 of disease 5 months after core decompression.

A, Coronal T1-weighted image (TR/TE, 450/20) of left hip before core decompression shows no focal bone marrow abnormality in femoral head.

B, Coronal T1-weighted image (450/20) obtained 5 months after core decompression shows newly developed hypointense signal line (arrows) of osteonecrosis between two drilling holes in femoral head. There was progressive change from stage 0 to stage 1 of disease.
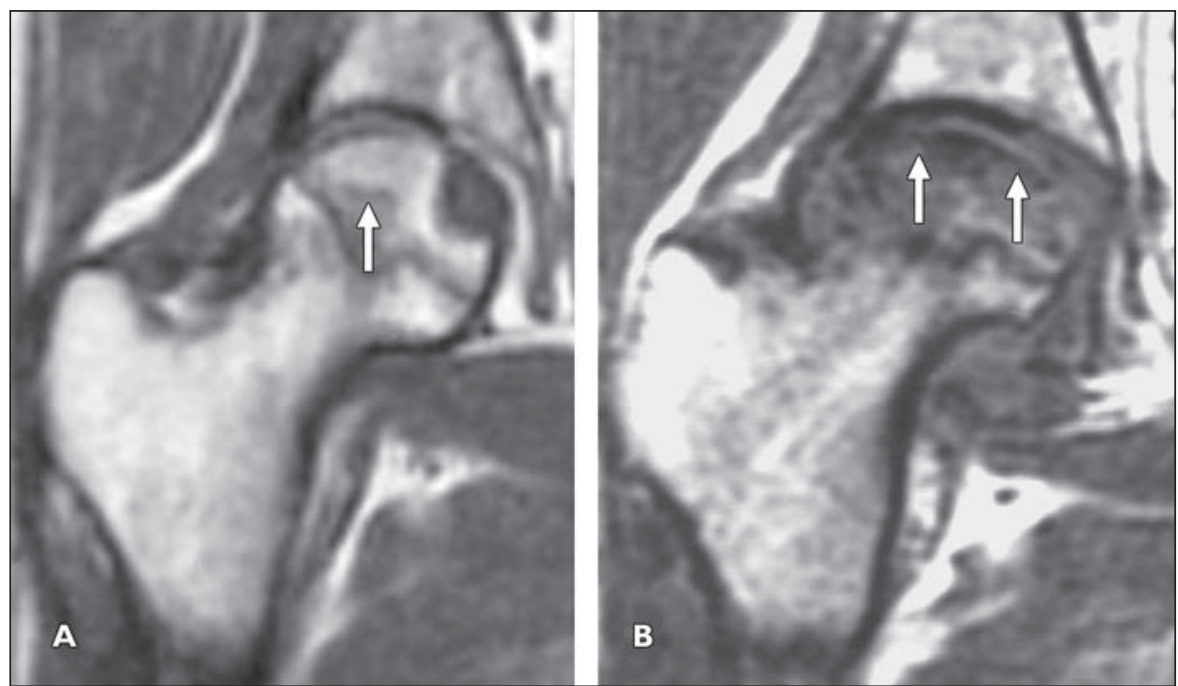

Fig. 4-44-year-old woman with osteonecrosis of femoral head with interval change from stage 1 to stage 2 of disease 9 months after core decompression of right hip.

A, Coronal T1-weighted image (TR/TE, 450/20) of right hip before core decompression shows hypointense line pattern (arrow) of osteonecrosis.

B, Coronal T1-weighted image (450/20) obtained 9 months after core decompression shows collapse of femoral head (arrows).

of the femoral head. This is because the acetabulum is supplied by different arteries and is drained by different veins from the proximal femur [27].

Our investigation has some limitations. The same patients in our small study were selected as the control group. Patients were studied at only one point in time and had had symptoms for various periods, up to 2 years. All patients undergoing core decompression may be subject to sampling error. There was no fat-saturation measurement with contrastenhanced images. A high-field magnet was not available during the study, and the use of a 0.5-T magnet may have constrained the signal-to-noise ratio, especially for the regions of interest in the hips studied. Although DCE-MRI can potentially assess hemodynamic changes, no histologic or angiographic analyses supported our DCE-MRI data.
In conclusion, intramedullary peak enhancement increased in the femoral head with progression of idiopathic osteonecrosis of the femoral head, whereas there was delayed peak enhancement in the femoral head in grade 0 hips and intertrochanteric stasis in advanced osteonecrosis of the femoral head, as evaluated by DCE-MRI. Such perfusion changes as shown on MRI can occur with early osteonecrosis in the absence of other MRI abnormalities.

\section{References}

1. Ficat RP, Arlet J. Necrosis of the femoral head. In: Hungerford DS, ed. Ischemia and necrosis of bone. Baltimore, MD: Williams \& Wilkins, 1980:11-52

2. Ficat RP. Idiopathic bone osteonecrosis of the femoral head: early diagnosis and treatment. $J$ Bone Joint Surg Br 1985; 67:3-9

3. Cova M, Kang YS, Tsukamoto H, et al. Bone marrow perfusion evaluated with gadolinium-enhanced dynamic fast MR imaging in a dog model. Radiology 1991; 179:535-539

4. Brody AS, Strong M, Babikian G, Sweet DE, Seidel FG, Kuhn JP. Avascular necrosis: early MR imaging and histologic findings in a canine model. AJR 1991; 157:341-345

5. Nadel SN, Debatin JF, Richardson WJ, et al. Detection of acute avascular necrosis of the femoral head in dogs: dynamic contrast-enhanced MR imaging vs. spin-echo and STIR sequences. AJR 1992; 159:1255-1261

6. Menezes NM, Connolly SA, Shapiro F, et al. Early ischemia in growing piglet skeleton: MR diffusion and perfusion imaging. Radiology 2007; 242:129-136

7. Bluemke DA, Petri M, Zerhouni EA. Femoral head perfusion and composition: MR imaging and spectroscopic evaluation of patients with systemic lupus erythematosus and at risk for avascular necrosis. Radiology 1995; 197:433-438

8. Malizos KN, Zibis AH, Dailiana Z, Hantes M, Karachalios T, Karantanas AH. MR imaging findings in transient osteoporosis of the hip. Eur $J$ Radiol 2004; 50:238-244

9. Aaron RK, Dyke JP, Ciombor DM, et al. Perfusion abnormalities in subchondral bone associated with marrow edema, osteoarthritis, and avascular necrosis. Ann N Y Acad Sci 2007; 1117:124-137

10. Mitchell DG, Rao VM, Dalinka MK, et al. Hematopoietic and fatty bone marrow distribution in the normal and ischemic hip: new observations with 1.5-T MR imaging. Radiology 1986; 161:199-202

11. Beltran J, Herman LJ, Burk JM, et al. Femoral head avascular necrosis: MR imaging with clinical-pathologic and radionuclide correlation. Radiology 1988; 166:215-220 


\section{Dynamic Contrast-Enhanced MRI of Femoral Idiopathic Osteonecrosis}

12. Huang GS, Chan WP, Chang YC, Chang CY, Chen CY, Yu JS. MR imaging of bone marrow edema and joint effusion in patients with osteonecrosis of the femoral head: relationship to pain. AJR 2003; 181:545-549

13. Turner DA, Templeton AC, Selzer PM, Rosenberg AG, Petasnick JP. Femoral capital osteonecrosis: MR finding of diffuse marrow abnormalities without focal lesions. Radiology 1989; 171:135-140

14. Brix G, Semmler W, Port R, et al. Pharmacokinetic parameters in CNS Gd-DTPA enhanced MR imaging. J Comput Assist Tomogr 1991; 15:621-628

15. Koo KH, Ahn IO, Song HR, Kim SY, Jones JP Jr. Increased perfusion of the femoral head in transient bone marrow edema syndrome. Clin Orthop Relat Res 2002; 402:171-175

16. Yamano K, Atsumi T, Muraki M. Transient osteonecrosis of the hip: what is the initial event? ARCO Newslett 1996; 8:115-116

17. Simmons DJ, Daum WJ, Totty W, Murphy WA. Correlation of MRI images with histology in avascular necrosis in the hip: a preliminary study.
J Arthroplasty 1989; 4:7-14

18. Lang P, Jergesen HE, Moseley ME, Block JE, Chafetz NI, Genant HK. Avascular necrosis of the femoral head: high-field-strength MR imaging with histologic correlation. Radiology 1988; 169: 517-524

19. Sakai T, Sugano N, Nishii T, Haraguchi K, Ochi T, Ohzono K. MR findings of necrotic lesions and the extralesional area of osteonecrosis of the femoral head. Skeletal Radiol 2000; 29:133-141

20. Vande Berg BE, Malghem JJ, Labaisse MA, Noel HM, Maldague BE. MR imaging of avascular necrosis and transient marrow edema of the femoral head. RadioGraphics 1993; 13:501-520

21. Shih TT, Liu HC, Chang CJ, et al. Correlation of MR lumbar spine bone marrow perfusion with bone mineral density in female subjects. Radiology 2004; 233:121-128

22. Tsuji T, Sugano N, Sakai T, Yoshikawa H. Evaluation of femoral perfusion in a non-traumatic rabbit osteonecrosis model with $\mathrm{T} 2 *$-weighted dynamic MRI. J Orthop Res 2003; 21:341-351
23. Ricci C, Cova M, Kang YS, et al. Normal age-related patterns of cellular and fatty bone marrow distribution in the axial skeleton: MR imaging study. Radiology 1990; 177:83-88

24. Mitchell DG, Kressel HY, Arger PH, Dalinka M, Spritzer CE, Steinberg ME. Avascular necrosis of the femoral head: morphology assessment by MR imaging with CT correlation. Radiology 1986; 161:739-742

25. Vande Berg BC, Malghem J, Lecourvet FE, Devogelaer J-P, Maldague B, Houssiau FA. Fat conversion of femoral marrow in glucocorticoid-treated patients: a cross-sectional and longitudinal study with magnetic resonance imaging. Arthritis Rheum 1999; 42:1405-1411

26. Chen WT, Shih TT, Chen RC, et al. Vertebral bone marrow perfusion evaluated with dynamic contrast-enhanced MR imaging: significance of aging and sex. Radiology 2001; 220:213-218

27. Ficat RP, Arlet J. Necrosis of the femoral head. In: Hungerford DS, ed. Ischemia and necrosis of bone. Baltimore, MD: Williams \& Wilkins, 1980:2-5

\section{F OR YOUR INFORMATION}

The American Roentgen Ray Society now provides instant Web exclusive access to its annual meeting abstracts. The abstracts, featured as a supplement to the American Journal of Roentgenology, summarize the latest comprehensive and clinically important information presented at ARRS's annual meetings. The abstracts can be viewed online by visiting www.ajronline.org. 